Wakker, Peter P. (1991), "Additive Representations of Preferences, A New Foundation of Decision Analysis; The Algebraic Approach." In Jean-Paul Doignon \& Jean-Claude Falmagne (Eds.), Mathematical Psychology: Current Developments, Springer, Berlin, 71-87.

Hereafter follows the published version of the paper. 


\title{
Additive Representations of Preferences, A New Foundation of Decision Analysis; The Algebraic Approach
}

\author{
Peter Wakker* \\ University of Nijmegen (NICI)
}

\begin{abstract}
In Wakker (1989b, 'Additive Representations of Preferences, A New Foundation of Decision Analysis'), a new foundation of decision analysis was given. The main tool was a way to derive comparisons of 'tradeoffs' from ordinal preferences, with comparisons of tradeoffs revealing orderings of utility differences. These comparisons of tradeoffs underly the construction of standard sequences in conjoint measurement theory. The restrictive structural assumption (every approach has its restrictive structural assumption) was of a topological nature, requiring continuity. This paper adapts the main results of Wakker (1989b) to the algebraic approach, where a solvability condition is required which is less restrictive than continuity.
\end{abstract}

\section{History, Motivation and Preview}

The foundations of decision analysis were for the major part laid around the fifties. The main results are, a.o., von Neumann \& Morgenstern (1944), Savage (1954), Suppes (1956), Anscombe \& Aumann (1963). An early brilliant contribution of Ramsey (1931) became appreciated and known only long after its appearance. It has never been fully understood: to the best of our knowledge there is no set-up in which all operations of Ramsey (1931) are formally correct. Also the contribution of de Finetti (1931, refined in de Finetti, 1937, 1974) has been underestimated, and misunderstood. While the derivation of von Neumann \& Morgenstern assumes probabilities given in advance, the derivation of de Finetti can be seen to assume utilities given in advance. Also the approach of Anscombe \& Aumann, as well as that of Pratt, Raiffa, \& Schlaifer (1964), assume not all, but still many, probabilities given. The derivation of Ramsey (1931) assumes probabilities $1 / 2$ given, through the 'ethically neutral' event; it is not a real characterization fully in terms of preferences.

The idea of conjoint measurement is to take several quantities to be measured as unknown, and to derive them jointly from observations. This idea was not commonly known around the fifties, when most of the foundations of decision analysis were laid. Only in the late sixties, through the work gathered in Krantz, Luce, Suppes, \& Tversky (1971), hereafter abbreviated KLST, did this idea become full-blown. When applied to decision analysis, it leads to the question of how to characterize/elicit both utilities and probabilities from

*The research has been made possible by a fellowship of the Royal Netherlands Academy of Arts and Sciences, and a fellowship of the Netherlands Organization for Scientific Research. Two anonymous referees made helpful comments. Please send all correspondence to: Peter Wakker, University of Nijmegen (NICI), P.O. Box 9104, 6500 HE Nijmegen, The Netherlands. 
preferences. Savage did raise that question, and solved it; this has finally led to the famous result in the first five chapters of Savage (1954). ${ }^{1}$ It can be argued that also Ramsey (1931) came close to the joint derivation of probabilities and utilities. Given the importance of subjective probabilities as the, paradoxical, operationalization of 'knowing what one doesn't know', and their importance as the refined modeling of degrees of knowledge, given the originality of using a conjoint measurement approach, given the well-written presentation, and given the high level of mathematics, the first five chapters of Savage (1954) constitute one of the most impressive scientific achievements of this century. The idea of conjoint measurement is well established nowadays and, mainly through KLST, its techniques are well-developed. Wakker (1989b), hereafter abbreviated [W], uses these new techniques to revisit the foundations of decision analysis of the fifties, and to obtain a new foundation hoped to contribute to those obtained around the fifties. The basic idea is to use 'tradeoffs' between dimensions (a dimension for each state of nature) to build up standard sequences on each dimension, thus eliciting utility. This is achieved by 'derived comparisons of tradeoffs'. Then the number of units of utility contingent on one state of nature that outweighs one unit of utility contingent on another state of nature gives (proportions of) probabilities.

The motivation to deviate from the approaches of von Neumann \& Morgenstern (1944), Anscombe \& Aumann (1963), Pratt, Raiffa, \& Schlaifer (1964), and to a lesser degree from Ramsey (1931) and Suppes (1956), is to avoid the necessity of probabilities given in advance. The motivation to deviate from Savage's set-up is that Savage needs to impose richness conditions on the state space through his postulate P6 and his nontransparent P7 (P7 is only transparent in those finite cases where it is implied by and does not contribute to the other axioms), and that Savage's axioms turned out to imply boundedness of utility in an unforeseen way; see Fishburn (1970, Section 14.1), or the second edition of 1972 of Savage (1954), footnote on p. 80. An extension of Savage's approach to unbounded utility has been given in Wakker (1989c, Theorem 33).

The motivation to deviate from de Finetti (1931) is, firstly, to allow for nonlinearity of utility, and, secondly, to allow for nonquantitative consequences for which linearity and de Finetti's coherence condition cannot even be defined. The starting question of our research was how to generalize de Finetti's 'Dutch book making', and has indeed led to the book [W]. It combines Savage's possibility to have nonlinear utility with de Finetti's possibility to have general, possibly finite, possibly infinite, state spaces. See Theorem 5 below. Instead of taking the state space as a continuum, as is basically done in Savage's set-up, we take the consequence space as a continuum (to be generalized somewhat in this paper). The latter is more customary in economic models, and in conjoint measurement as considered in the present paper. The present paper shows how the restrictive continuity assumption of [W] can be replaced by the, according to Wakker (1988) more general and intuitively preferable, restrictive solvability axiom, together with the nonrestrictive and merely technical Archimedean axiom. Thus it gives a survey and generalization of the results in [W]. For extensive motivations and elucidations see [W]. The sequel of this paper will only now and then supplement additional motivation or history. A self-contained presentation of all proofs below would take much space, so instead we often refer to the proofs and reasonings in other papers by the author, mainly [W] and Wakker (1990c), and show how these should be adapted.

Section 2 starts with a presentation of de Finetti's theorem on $\mathbb{R}^{n}$. Section 3 sketches the set-up of additive conjoint measurement on a Cartesian product $\times_{i=1}^{n} \mathcal{C}_{i}$. Here the 'derived comparisons of tradeoffs' formulation is used to obtain an alternative, and we hope

\footnotetext{
${ }^{1}$ Section 3.3.3 in von Neumann \& Morgenstern (1944), footnote 2, does announce the idea of conjoint measurement for subjective expected utility.
} 
transparent, formulation for standard additive representation results. Section 4 gives a characterization of subjective expected utility (SEU) maximization on a Cartesian product $\mathcal{C}^{n}$, i.e., obtains the desired generalization of de Finetti's theorem. Nowadays deviations from SEU are popular; Section 5 characterizes such a deviation, i.e., Choquet-expected utility, which allows probabilities to be nonadditive. Again the domain is a Cartesian product $\mathcal{C}^{n}$. Nakamura (1990a,b) has recently also obtained characterizations of SEU and CEU under the algebraic assumptions, with a solvability axiom that is somewhat stronger than the one in KLST and in this paper. His solvability axiom implies the existence of certainty equivalents and rules out the equally spaced case. The main tool is a bi/multi symmetry axiom in the spirit of Pfanzagl (1968) and KLST (Section 6.9), instead of the tool of derived tradeoffs. Finally some basic definitions are given. A function $V$ represents a binary relation on its domain if for all arguments $x, y$,

$$
x \succeq y \Leftrightarrow V(x) \geq V(y) .
$$

If there exists a representing function for a binary relation $\succeq$ then the binary relation is a weak order, i.e., it is transitive $(\forall x, y, z:[x \succeq y, y \succeq z] \Rightarrow[x \succeq z])$ and complete $(\forall x, y: x \succeq y$ or $y \succeq x)$.

\section{Digression; The Theorem of De Finetti}

This section presents a short sketch of one version of de Finetti's theorem, surely not the most general version. For references to proofs see [W, Section A2]. De Finetti's result can be seen to imply linearity of utility, a condition that deters economists. In many places in the literature authors have presented de Finetti's results in a misleading way, by suggesting that 'incoherence' is identical to violation of monotonicity ('taking a sure loss'), and by putting under the cover the nonselfevident linear operations (only additivity in our presentation below with continuity presupposed) as needed in the book making process to lead incoherent preferences into a sure loss.

Let $\succeq$ be a binary relation on $\mathbb{R}^{n}$. For an element $x$ of $\mathbb{R}^{n}, x_{i}$ denotes its $i$ th coordinate, $i=1, \ldots, n$. $\succeq$ is strongly monotonic if $x \succ y$ whenever $x \neq y$ and $x_{i} \geq y_{i}$ for all $i$. It is continuous if a first alternative is weakly preferred to a second alternative whenever the first alternative can be approximated by a first sequence of alternatives, and the second alternative by a second sequence, such that each $i$ th term of the first sequence is weakly preferred to the $i$ th term of the second. The crucial condition in the theorem below is 'additivity', i.e., 'zeroindependence' in the terminology of Moulin (1988). We call $\succeq$ additive if $x \succeq y$ implies $x+v \succeq y+v$, for all $x, y, v$.

THEOREM 1 Let $\succeq$ be a binary relation on $\mathbb{R}^{n}$. Then the following two statements are equivalent:

(i) There exist positive numbers $p_{1}, \ldots, p_{n}$, summing to one, such that $x \mapsto$ $\sum_{i=1}^{n} p_{i} x_{i}$ represents $\succeq$.

(ii) The binary relation $\succeq$ is a continuous strongly monotonic additive weak order.

The numbers $p_{i}$ in Statement (i) above are uniquely determined. 
De Finetti applied the above theorem to decision making under uncertainty. Suppose an asymmetric die with $n$ sides will be thrown, and $x \in \mathbb{R}^{n}$ denotes a gamble that yields $\$ x_{j}$ if side $j$ will come up. Because a decision maker with preference relation $\succeq$ over gambles is uncertain about the side that will come up, he is uncertain about the amount of money $x_{j}$ that will result from the gamble $x$. The above theorem has characterized expected value maximization. Actually de Finetti obtained a more general result, where the domain of the preference relation can be a subset of $I R^{n}$, as long as for every gamble there is a 'fair price', i.e., a constant gamble that is equivalent. In presentations of de Finetti's result the additivity condition is usually not discussed, it is implicit in a book making set-up ${ }^{2}$ together with a multiplication condition that was implied above by additivity and continuity, and the role of strong monotonicity (or somewhat weaker versions thereof) is overemphasized. Because of the book making set-up, some may disagree with our claim that the above theorem describes what de Finetti proved. Anyway, the aim to generalize the above theorem to nonlinear utility has initiated our research. The major step forward was our coincidental discovery of the book KLST, and the theory of additive conjoint measurement presented there.

\section{Additive Representation through Comparisons of Tradeoffs and Contradictory Tradeoffs}

This section presents the results of additive representation in a version somewhat deviating from KLST.

\subsection{Elementary Definitions of Additive Conjoint Measurement}

Let $\times_{i=1}^{n} \mathcal{C}_{i}$ be a Cartesian product of nonempty sets. Its elements are called alternatives. Sections 4 and 5 consider identical coordinate sets $\mathcal{C}_{i}$, i.e., $\forall i: \mathcal{C}_{i}=\mathcal{C}$ for some space $\mathcal{C}$. By $\succeq$ we denote a binary (preference) relation on $\times_{i=1}^{n} \mathcal{C}_{i} ; \succ, \preceq, \prec$, and $\sim$ are as usual. A function $V$ is additive if

$$
V: x \mapsto \sum_{j=1}^{n} V_{j}\left(x_{j}\right)
$$

for some functions $V_{j}: \mathcal{C}_{j} \rightarrow \mathbb{R}, j=1, \ldots, n$. If an additive function represents $\succeq$, then the $V_{j}$ 's are called additive value functions. A function $V$ is an interval scale (or 'cardinal') if, loosely speaking, it 'can be replaced' by any function $\tau+\sigma V$ for real $\tau$ and positive $\sigma$. If an additive function is an interval scale, then the additive value functions are 'joint interval scales', i.e., arbitrary real numbers ('locations') can be added to each of them, and they can be multiplied by a joint positive factor ('scale'). These two uniqueness results are equivalent, we shall usually make explicit the result applying to the additive function.

We write $x_{-i} v_{i}$ for $x$ with $x_{i}$ replaced by $v_{i}$, and, for $i \neq j$, we write $x_{-i, j} v_{i}, w_{j}$ for $x$ with $x_{i}$ replaced by $v_{i}, x_{j}$ replaced by $w_{j}$. Coordinate $i$ is inessential (with respect to $\succeq)$ if $\left[\forall x, y_{i}: x \sim x_{-i} y_{i}\right]$. The opposite of inessential is essential. $\succeq$ satisfies restricted solvability if:

$$
x_{-i} a_{i} \succ y \succ x_{-i} c_{i} \Rightarrow \exists b_{i}: x_{-i} b_{i} \sim y
$$

for all alternatives $x_{-i} a_{i}, y, x_{-i} c_{i}$.

\footnotetext{
${ }^{2}$ There a decision maker/bookmaker, considering some gambles (acts with as consequences amounts of money) as 'fair' (equivalent to the 0-gamble), should also consider all linear combinations of these as fair.
} 
We say $\succeq$ satisfies independence of equal coordinates, or coordinate independence (CI) for short, if, for all alternatives in question,

$$
\left[x_{-i} v_{i} \succeq y_{-i} v_{i}\right] \Leftrightarrow\left[x_{-i} w_{i} \succeq y_{-i} w_{i}\right]
$$

In the usual way $\succeq_{i}$ is defined for each coordinate $i$, i.e., $v_{i} \succeq_{i} w_{i}$ if and only if there exists $x$ such that $x_{-i} v_{i} \succeq x_{-i} w_{i}$. Similarly $\succ_{i}, \preceq_{i}, \prec_{i}, \sim_{i}$ can be derived from $\succ, \preceq, \prec, \sim$. The following implications of CI are straightforward. Under CI, $v_{i} \succeq_{i} w_{i}$ holds if and only if $y_{-i} v_{i} \succeq y_{-i} w_{i}$ for all $y$. Also under CI it follows that if $\succeq$ is a weak order, then so is $\succeq_{i}$, the relations $\succ_{i}, \preceq_{i}, \prec_{i}, \sim_{i}$ can be derived from $\succeq_{i}$ in the usual way, and we have the following monotonicities:

$$
\begin{aligned}
{\left[\forall i: x_{i} \succeq_{i} y_{i}\right] } & \Rightarrow[x \succeq y] \\
{\left[\forall i: x_{i} \succeq_{i} y_{i} \text { and } \exists j: x_{j} \succ_{j} y_{j}\right] } & \Rightarrow[x \succ y] ; \\
{\left[\forall i: x_{i} \sim_{i} y_{i}\right] } & \Rightarrow[x \sim y]
\end{aligned}
$$

Next the Archimedean axiom is defined. For $n=2$ the definition below coincides with that of KLST. There are several, for additive representations equivalent, ways to extend this condition to three or more dimensions; KLST do not make explicit which one they have in mind. One way we adopt below. An alternative would be to take in the definition below equivalences $x_{-j} z_{j}^{k} \sim y_{-j} z_{j}^{k+1}$ for all $k=1,2, \ldots$, instead of the special case with $x=x_{-i} v_{i}, y=x_{-i} w_{i}$ as adopted below. It can be seen that for all purposes this version of the Archimedean axiom is equivalent to our choice. For coordinate $j$ we call $z_{j}^{1}, z_{j}^{2}, \ldots$ a standard sequence (on coordinate $j$, with respect to $\succeq$ ), if there exist $x, i \neq j$, and $v_{i} \chi_{i} w_{i} \in \mathcal{C}_{i}$, such that $x_{-i, j} v_{i}, z_{j}^{k} \sim x_{-i, j} w_{i}, z_{j}^{k+1}$ for all $k=1,2, \ldots$. A standard sequence may be infinite or finite of any length. A standard sequence is bounded if there exist $z_{j}^{\text {sup }}$ and $z_{j}^{\min }$ such that $z_{j}^{\text {sup }} \succeq_{j} z_{j}^{k} \succeq_{j} z_{j}^{\text {min }}$ for all $k$. The Archimedean axiom requires that every bounded standard sequence be finite.

\subsection{Comparisons of Tradeoffs and Contradictory Tradeoffs}

Now the method to compare tradeoffs is introduced. It is the central tool in most of the author's works, and uses the tradeoff idea from multiattribute utility theory. [W, reference footnote rf13 on p. 168] refers to several related ideas in the literature. Still, the simplicity and generality of results that can be obtained through its use, to our knowledge have not been observed before. Below, a pair $x_{i} y_{i}$ may be interpreted as the 'tradeoff' of receiving $x_{i}$ instead of $y_{i}$, or as the strength of preference of $x_{i}$ over $y_{i}$. We write, for $x_{i}, y_{i}, v_{i}, w_{i} \in \mathcal{C}_{i}$, $x_{i} y_{i} \succ_{i}^{* *} v_{i} w_{i}$ if:

there exist alternatives $a, b$ such that

$$
\begin{aligned}
a_{-i} x_{i} & \succeq b_{-i} y_{i} \text { and } \\
\text { not } a_{-i} v_{i} & \succeq b_{-i} w_{i} .
\end{aligned}
$$

The idea is that the above preferences reveal that the 'tradeoff' of getting $x_{i}$ instead of $y_{i}$ is better than the tradeoff of getting $v_{i}$ instead of $w_{i}$. Note that the notation $\succ_{i}^{* *}$ 'remembers' the coordinate $i$, but 'forgets' the alternatives $a, b$. The notation is misleading in contexts 
where this forgetting of alternatives is not appropriate, and where it then leads to a kind of contradiction. Theorem 3 shows that the notation is appropriate if and only if the preference relation is additively representable, given the other conditions there. After the definition of $\succeq_{i}^{*}$, we shall describe the kind of contradiction that may result for general preferences.

We write $\succeq_{i}^{*}$ instead of $\succ_{i}^{* *}$ if in (6) we have ' $\preceq$ ' instead of 'not $\succeq$ '. The reason for two ${ }^{* *}$ 's in $\succ_{i}^{* *}$, and only one ${ }^{*}$ in $\succeq_{i}^{*}$, is to avoid the misunderstanding that in general $\succ_{i}^{* *}$ would be the asymmetric part of $\succeq_{i}^{*}$. For general preference relations $\succ_{i}^{* *}$ does not have to be the asymmetric part of $\succeq_{i}^{*}$. One easily verifies by substitution:

LEMma 2 If there exist additive value functions $\left(V_{j}\right)_{j=1}^{n}$ for $\succeq$, then:

$$
\begin{gathered}
x_{i} y_{i} \succ_{i}^{* *} v_{i} w_{i} \Rightarrow V_{i}\left(x_{i}\right)-V_{i}\left(y_{i}\right)>V_{i}\left(v_{i}\right)-V_{i}\left(w_{i}\right) \\
\text { and } \\
x_{i} y_{i} \succeq_{i}^{*} v_{i} w_{i} \Rightarrow V_{i}\left(x_{i}\right)-V_{i}\left(y_{i}\right) \geq V_{i}\left(v_{i}\right)-V_{i}\left(w_{i}\right) .
\end{gathered}
$$

Coordinate $i$ reveals contradictory (orderings of) tradeoffs if there exist $x_{i}, y_{i}, v_{i}, w_{i}$ such that both

$$
\left[x_{i} y_{i} \succeq_{i}^{*} v_{i} w_{i}\right] \text { and }\left[v_{i} w_{i} \succ_{i}^{* *} x_{i} y_{i}\right] \text {. }
$$

By the previous lemma contradictory tradeoffs clearly falsify additive representability. Under the conditions of Theorem 3 absence of contradictory tradeoffs will not only be necessary, but also sufficient for additive representability. The condition that no coordinate reveals contradictory tradeoffs, when formulated entirely in terms of preferences, can, with an interchange of symbols as compared to the above notation, be reformulated as:

$$
\begin{array}{llll} 
& x_{-i} a_{i} \preceq y_{-i} b_{i} & \& \quad v_{-i} a_{i} \succeq w_{-i} b_{i} \\
x_{-i} c_{i} & \succeq y_{-i} d_{i} & \Rightarrow \quad & \\
& & v_{-i} c_{i} \succeq w_{-i} d_{i}
\end{array}
$$

for all $i, x, \ldots, d_{i}$.

For $n=2$ the above condition is identical to 'triple cancellation' of KLST, hence it is called generalized triple cancellation. It is easily seen that generalized triple cancellation, hence the absence of contradictory tradeoffs revealed by coordinates, implies CI if $\succeq$ is reflexive (set $a_{i}=b_{i}, c_{i}=d_{i}, x=y$ ). This leads to the main theorem on additive representation.

TheOREM 3 Let $\succeq$ be a binary relation on a Cartesian product $\times_{i=1}^{n} \mathcal{C}_{i}$. Suppose at least two coordinates are essential, and restricted solvability is satisfied. Then the following three statements are equivalent:

(i) There exists an additive representation for $\succeq$.

(ii) The binary relation $\succeq$ is a weak order, it satisfies the Archimedean axiom, and no coordinate reveals contradictory tradeoffs.

(iii) The binary relation $\succeq$ is a weak order that satisfies the Archimedean axiom and generalized triple cancellation. 
If at least three coordinates are essential, then the absence of contradictory tradeoffs in Statement (ii), respectively generalized triple cancellation in Statement (iii), can be weakened to $C I$.

Further, the additive representation in (i) is an interval scale.

PROOF. [W] gives a proof from the scratch for the above theorem in the context of the topological approach. In this proof an essential step is to construct, loosely speaking, midpoints between coordinates (see [W, Stage 5]). In the present more general set-up of KLST, this no longer is possible, as pointed out in [W, end of Section III.6]. So we sketch an alternative proof. Actually, the above theorem is just a reformulation and regrouping of results already present in KLST. For the case of three or more essential coordinates the result follows mainly from Theorem 6.13 of KLST, and the observation that generalized triple cancellation, so the absence of contradictory tradeoffs by a coordinate as well, implies CI. For the case of exactly two essential coordinates, the theorem follows from the observation that generalized triple cancellation coincides (after suppression of the inessential coordinates) with triple cancellation of KLST, and the end of Section 6.2.4 in KLST. As usual, uniqueness results are standard.

Statements (ii) and (iii), contrary to the formulation in KLST, do not separate between the case of two essential coordinates, and the case of three or more essential coordinates. In a mathematical sense this 'achievement' is no surprise: For the case of two essential coordinates the conditions are equivalent to those used by KLST, for the case of three or more essential coordinates they are simply stronger than CI as used by KLST. Still there is interest in a unified formulation, even if logically weaker, as we have learned from experience. Let us also mention Wakker (1991), where it is shown that for the 'equally spaced' case the proof and conditions of the above theorem can be considerably simplified; the only implications of CI or generalized triple cancellation, needed for that case, are (3), (4), and (5) ('weak separability').

\section{Subjective Expected Utility Through Comparisons of Tradeoffs and Contra- dictory Tradeoffs}

This section characterizes subjective expected utility (SEU) maximization, i.e., the representation in Statement (i) in Theorem 5. The set-up of decision making under uncertainty as described at the end of Section 2 is adopted. This comes down to conjoint measurement with all coordinate sets $\mathcal{C}_{j}$ identical to one set $\mathcal{C}$. The following paragraph gives an interpretation.

Suppose there are $n$ 'states of nature', where exactly one is the true state, the other states are not true. A decision maker is uncertain about which of the states is the true one, and cannot change the truth of states. Further a set $\mathcal{C}$ of consequences is given, with elements denoted as $\alpha, \beta, \ldots$. An element $x$ from the $n$-fold Cartesian product $\mathcal{C}^{n}$ is called act rather than alternative, and is interpreted as a course of action or a decision alternative of the decision maker that will result in $x_{j}$ if the $j$ th state of nature is true. Because the decision maker does not know for sure which state is true, he or she does not know for sure which consequence will result from an act, and faces decision making under uncertainty. Often an act $x$ is considered a function assigning $x_{j}$ to the $j$ th state, instead of an $n$-tuple. Note that this set-up does not require (but neither excludes) that there are given 'objectively known' probabilities for the states of nature in advance. If such probabilities are given and contain all relevant information about the states of nature, then one speaks of decision making under risk. 
We write $\alpha \beta \succ^{* *} \gamma \delta$ if there exists an $i$ such that $\alpha \beta \succ_{i}^{* *} \gamma \delta$, i.e., there exist acts $a, b$ and a state $i$ such that

$$
\begin{aligned}
a_{-i} \alpha & \succeq b_{-i} \beta \text { and } \\
\text { not } a_{-i} \gamma & \succeq b_{-i} \delta .
\end{aligned}
$$

Note that, as compared to (6), not only the acts $a, b$ are 'forgotten', but also the state $i$, in the notation $\succ^{* *}$. The notation will be misleading in contexts where this forgetting of the acts and state is not appropriate, and where this leads to a kind of contradiction. Theorem 5 will show that the notation is appropriate if and only if the preference relation is representable by subjective expected utility, given the other conditions there. For inessential states $i$ we have $\alpha \beta \succeq_{i}^{*} \gamma \delta$ for all $\alpha, \beta, \gamma, \delta$, which is not informative. Hence we define $\alpha \beta \succeq^{*} \gamma \delta$ if in (10) above we have ' $\preceq$ ' instead of 'not $\succeq$ ', and further state $i$ is essential. One easily verifies by substitution:

Lemma 4 If SEU holds, i.e., Statement (i) in Theorem 5 holds, then:

$$
\begin{gathered}
\alpha \beta \succ^{* *} \gamma \delta \Rightarrow U(\alpha)-U(\beta)>U(\gamma)-U(\delta) \\
\quad \text { and } \\
\alpha \beta \succeq^{*} \gamma \delta \Rightarrow U(\alpha)-U(\beta) \geq U(\gamma)-U(\delta) .
\end{gathered}
$$

Again, the following kind of contradiction is to be excluded: $\succeq$ reveals contradictory (orderings of) tradeoffs (on consequences) if there exist consequences $\alpha, \beta, \gamma, \delta$ such that both

$$
\left[\alpha \beta \succeq^{*} \gamma \delta\right] \text { and }\left[\gamma \delta \succ^{* *} \alpha \beta\right] \text {. }
$$

Obviously the revelation of contradictory orderings of tradeoffs on consequences falsifies SEU. Theorem 5 will show that, under the usual conditions as supposed there, the absence of contradictory tradeoffs on consequences is not only necessary, but also sufficient, for SEU. In terms of the relations $\succeq_{i}^{*}$ and $\succ_{i}^{* *}$ as introduced in Section $3, \succeq$ reveals contradictory tradeoffs (on consequences) if there exist $\alpha, \beta, \gamma, \delta$, and a $j$ th state and an essential $i$ th state, such that both

$$
\left[\alpha \beta \succeq_{i}^{*} \gamma \delta\right] \text { and }\left[\gamma \delta \succ_{j}^{* *} \alpha \beta\right] .
$$

Note that the $\succ_{j}^{* *}$ relation above automatically implies essentiality of the $j$ th state. This shows how the present condition strengthens the condition of absence of contradictory tradeoffs revealed by coordinates/states, as considered in Section 3. Now also orderings of differences contingent on different states should not yield contradicts. This is typically the way in which SEU restricts additive representability: the orderings of differences should be the same for different states, leading to proportionality of the additive value functions (given common zero), thus to subjective expected utility. Finally the condition of absence of contradictory tradeoffs on consequences is presented entirely in terms of preferences. This shows most clearly its direct empirical content, and the way to test it; intuitively it is less transparent, 
we think. Again the restriction is added that state $i$ is essential. The condition below has been called 'cardinal coordinate independence' in earlier writings by the author. Wakker (1984) refers to Axiom 5 in Section 8.2.6 of KLST as the condition in the literature most similar to cardinal coordinate independence.

$$
\begin{array}{llll}
x_{-i} \alpha \preceq y_{-i} \beta & \& & v_{-j} \alpha \succeq w_{-j} \beta \\
& x_{-i} \gamma \succeq y_{-i} \delta & \Rightarrow & \\
& & v_{-j} \gamma \succeq w_{-j} \delta
\end{array}
$$

for all essential states $i$, and $x, \ldots, \delta$.

A related work, not known to the author when writing [W], is Fishburn (1990), who gives a nontransitive extension of the result below for the topological approach. It can be seen that each of his Axioms 4 (which is very close to cardinal coordinate independence when restricted to the transitive case) and 5 would, in addition to additive representability, give SEU.

Theorem 5 Let $\succeq$ be a binary relation on a Cartesian product $\mathcal{C}^{n}$. Suppose at least two states are essential, and restricted solvability is satisfied. Then the following two statements are equivalent:

(i) There exist nonnegative $\left(p_{j}\right)_{j=1}^{n}$, summing to one, and a function $U: \mathcal{C} \rightarrow \mathbb{R}$ such that $x \mapsto \sum_{j=1}^{n} p_{j} U\left(x_{j}\right)$ represents $\succeq$.

(ii) The binary relation $\succeq$ is a weak order, satisfies the Archimedean axiom, and does not reveal contradictory tradeoffs.

Further, the $p_{i}$ 's are uniquely determined, and $U$ is an interval scale.

PROOF. Again the proof of Theorem IV.2.7 of [W] cannot be invoked because no continuity is available. So again the proof is based on results from KLST. It is straightforwardly seen that the absence of contradictory tradeoffs, revealed by $\succeq$, implies that no state/coordinate can reveal contradictory tradeoffs; this is direct both for essential states, and for inessential states. So by Theorem 3 there exist additive value functions $\left(V_{j}\right)_{j=1}^{n}$ for $\succeq$. Showing that the additive value functions are proportional, after a choice of a common zero, can be done exactly as at the end of the proof of Theorem 2.1 of Wakker (1984). As usual, uniqueness results are standard.

\section{Choquet-Expected Utility with Nonadditive Probabilities through Compar- isons of Tradeoffs and Contradictory Tradeoffs}

In this section the study of decision making under uncertainty is continued. So again $\succeq$ is a preference relation on a Cartesian product $\mathcal{C}^{n}$. SEU is generalized by allowing probabilities to be nonadditive. This was initiated by Schmeidler (1989, first version 1982), and, building on that, by Gilboa (1987). The formal term for nonadditive probability, in want for a better term, is 'capacity'. An alternative term might be 'cumulative belief function'. This term expresses the phenomenon that the essential aspect of this function is its values in 'cumulative events', i.e., events of receiving some consequence or anything better; this is apparent in Formula (16) below. The set $\{1, \ldots, n\}$ of coordinates is again taken as state space. A function $v: 2^{\{1, \ldots, n\}} \rightarrow[0,1]$ is a capacity if $v(\emptyset)=0, v(\{1, \ldots, n\})=1$, and $v$ is 
monotonic with respect to set-inclusion, i.e., $A \supset B \Rightarrow v(A) \geq v(B)$. So as compared to a probability distribution, additivity has been weakened to monotonicity. We say $\succeq$ maximizes Choquet-expected utility (CEU) if there exist a utility function $U: \mathcal{C} \rightarrow I R$ and a capacity $v$ such that the preference relation is represented by

$$
x \mapsto C E U(x)=\int_{\{1, \ldots, n\}}(U \circ x) d v
$$

where the integration with respect to the capacity $v$ of course needs elucidation and definition. In it, $x$ should be taken as a function from $\{1, \ldots, n\}$ to $\mathcal{C}$, assigning $x_{j}$ to each state $j$. The integral is taken as the 'Choquet integral', introduced in Choquet (1953-4). First the general definition is given, which can also be used for infinite state spaces. Next in (17) a more accessible formulation is given. $\mathrm{CEU}(x):=$

$$
\int_{\mathbb{R}^{+}} v\left(\left\{s \in\{1, \ldots, n\}: U\left(x_{s}\right) \geq \tau\right\}\right) d \tau+\int_{\mathbb{R}_{-}}\left[v\left(\left\{s \in\{1, \ldots, n\}: U\left(x_{s}\right) \geq \tau\right\}\right)-1\right] d \tau .
$$

In the present finite state case it can be rewritten as follows. Let the permutation $\pi$ describe a 'favorability ordering of the states in accordance with $x^{\prime}$, i.e., $x_{\pi(j)} \succeq x_{\pi(j+1)}$ for all $j$.

$$
C E U(x)=\sum_{j=1}^{n}[v(\{\pi(1), \ldots, \pi(j)\})-v(\{\pi(1), \ldots, \pi(j-1)\})] \times U\left(x_{\pi(j)}\right) .
$$

So, first the utilities $U\left(x_{j}\right)$ are ordered from best, $U\left(x_{\pi(1)}\right)$, to worst, $U\left(x_{\pi(n)}\right)$. Next to each utility $U\left(x_{\pi(j)}\right)$ the 'decision weight' $[v(\{\pi(1), \ldots, \pi(j)\})-v(\{\pi(1), \ldots, \pi(j-1)\})]$ is attached, i.e., the marginal contribution in capacity to the better states. In [W, Chapter VI] further elucidations are given.

The same way to evaluate acts was developed independently in Luce (1988), in his extension of the dual bilinear model to the case of more than two states of nature. The identity of his method with Choquet-expected utility may appear from comparison of his Formulas (7a), (7b), and (8) with [W]'s Formulas (VI.2.7) and (VI.2.11); Luce's Formula (8) is equivalent to Wakker's second part of Formula (VI.2.11). The set-up of Luce incorporates multi-stage gambles and operations on utility and gambles (acts), hence is different from ours.

As usual the binary relation $\succeq$ on $\mathcal{C}$ is derived from the binary relation $\succeq$ on $\mathcal{C}^{n}$ through constant acts. Under SEU this can be seen to coincide with all $\succeq_{i}$ 's for essential $i$. A set of acts is comonotonic if for no pair of elements $x, y$ from the set, and no states $i, j$, we both have $x_{i} \succ x_{j}$ and $y_{j} \succ y_{i}$. The importance of this condition can easily be understood from Formula 16: comonotonic acts have same cumulative events. We say ' $x$ and $y$ are comonotonic' if $\{x, y\}$ is. Intuition for comonotonicity can be inferred from Yaari (1987, p. 104), or Wakker (1990a, Example 1 up to Definition 3), or Wakker (1990b, Section 4). The largest comonotonic sets can be seen to be rank-ordered cones, i.e., sets of acts of the form $\left\{x \in \mathcal{C}^{n}: x_{\pi(1)} \succeq x_{\pi(2)} \succeq \ldots \succeq x_{\pi(n)}\right\}$, where $\pi$ is a permutation. Armstrong (1990) generalizes maximal comonotonic sets, rank-ordered subsets, and their relations, to more general spaces.

It can be seen that for all acts within one comonotonic set the permutation $\pi$ of Formula (17), and hence the decision weights $[v(\{\pi(1), \ldots, \pi(j)\})-v(\{\pi(1), \ldots, \pi(j-1)\})]$ there, can be taken the same. Hence within a comonotonic set CEU looks like usual SEU. Therefore the method to characterize CEU is to restrict conditions of usual expected utility to comonotonic subsets. The most important empirical implication of CEU may be 'comonotonic coordinate independence', the analogue of the most important empirical implication of SEU, Savage's P2 postulate of the sure-thing principle. Savage's P2 has been called CI in this paper. 
DEFINITION 6 We say that $\succeq$ satisfies comonotonic independence of equal coordinates, or comonotonic coordinate independence for short, abbreviated Com. CI, if (2) has to hold only if the two acts in question are comonotonic.

To the best of our knowledge this condition was first introduced in Wakker (1986). It is illustrated in Wakker (1989a). The condition adapts Schmeidler's condition of comonotonic independence with respect to probabilistic mixtures of 'lottery-acts' for decision making under risk to decision making under uncertainty, like Savage's sure-thing principle adapts the Marschak/Nash-independence condition for decision making under risk to decision making under uncertainty. Also, in exactly the same way as CI was adapted to CEU, the derived orderings of tradeoffs can be adapted to CEU. The subindex c below abbreviates 'comonotonic', indicating that the acts in question are comonotonic.

We write $\alpha \beta \succ_{c}^{* *} \gamma \delta$ if there exist acts exactly as in (10), but now comonotonic. One of the greatest difficulties in our first development of the theory, adapted in this section to the algebraic approach, was to find the right notion of essentiality of $i$ in the definition of $\succeq_{c}^{*}$ below. For a subset $E$ of acts, coordinate $i$ is inessential on $E$ if $\left[\forall x, x_{-i} y_{i} \in E: x \sim x_{-i} y_{i}\right]$. The opposite of inessential on $E$ is again essential on $E$. We define $\alpha \beta \succeq_{c}^{*} \gamma \delta$ if in (10) we have ' $\preceq$ ' instead of 'not $\succeq$ ', and state $i$ is essential on a comonotonic set containing all four acts in question. Obviously this implies that the four acts are comonotonic.

Note that, because of comonotonicity, $\pi$ in Formula (17) is the same for all four acts used in the definitions of $\succeq_{c}^{*}$ and $\succ_{c}^{* *}$. Also note that $v(\{\pi(1), \ldots, \pi(i)\})-v(\{\pi(1), \ldots, \pi(i-1)\})$ is positive because of essentiality of $i$. Thus one easily derives from Formula (17):

LEMma 7 If CEU holds then:

$$
\begin{gathered}
\alpha \beta \succ_{c}^{* *} \gamma \delta \Rightarrow U(\alpha)-U(\beta)>U(\gamma)-U(\delta) \\
\quad \text { and } \\
\alpha \beta \succeq_{c}^{*} \gamma \delta \Rightarrow U(\alpha)-U(\beta) \geq U(\gamma)-U(\delta) .
\end{gathered}
$$

We say that $\succeq$ reveals comonotonic-contradictory (orderings of) tradeoffs (on consequences) if there exist $\alpha, \beta, \gamma, \delta$ such that both

$$
\left[\alpha \beta \succeq_{c}^{*} \gamma \delta\right] \text { and }\left[\gamma \delta \succ_{c}^{* *} \alpha \beta\right] .
$$

Obviously the revelation of comonotonic-contradictory tradeoffs on consequences falsifies CEU. Theorem 8 will show that the absence of comonotonic-contradictory tradeoffs on consequences is not only necessary, but also sufficient, for CEU, given the conditions there. Next the condition of absence of comonotonic-contradictory tradeoffs on consequences is presented entirely in terms of preferences. Again the restriction is added that state $i$ is essential. The condition below has been called 'comonotonic cardinal coordinate independence' in earlier writings by the author:

$$
\begin{array}{llll}
x_{-i} \alpha \preceq y_{-i} \beta & \& & v_{-j} \alpha \succeq w_{-j} \beta \\
x_{-i} \gamma \succeq y_{-i} \delta & \Rightarrow & & \\
& & & v_{-j} \gamma \succeq w_{-j} \delta
\end{array}
$$

where $x_{-i} \alpha, y_{-i} \beta, x_{-i} \gamma, y_{-i} \delta$ are contained in a comonotonic set on which state $i$ is essential, and also $v_{-j} \alpha, w_{-j} \beta, v_{-j} \gamma, w_{-j} \delta$ are comonotonic. 
Finally a technical comment is given on the Archimedean axiom. In full generality the Archimedean axiom as formulated above for additive representations no more has to hold under Choquet-expected utility. The reason is that the condition $v_{i} \chi_{i} w_{i}$ is no more useful, now that additive representability no longer holds and the relation $\sim_{i}$ has different characteristics. So the 'comonotonic Archimedean axiom' is introduced. A 'comonotonic standard sequence' as defined next may be infinite or finite of any length. For coordinate $j$ we call $z_{j}^{1}, z_{j}^{2}, \ldots$ a comonotonic standard sequence (on coordinate $j$, with respect to $\succeq$ ), if there exist $x, i \neq j$, and $v_{i} \not w_{i} \in \mathcal{C}$, such that $x_{-i, j} v_{i}, z_{j}^{k} \sim x_{-i, j} w_{i}, z_{j}^{k+1}$ for all $k=1,2, \ldots$, and further all acts in question are contained in a comonotonic set on which $i$ is essential. A standard sequence is bounded if there exist $z_{j}^{\text {sup }}$ and $z_{j}^{\text {min }}$ such that $z_{j}^{\text {sup }} \succeq z_{j}^{k} \succeq z_{j}^{\min }$ for all $k$. The comonotonic Archimedean axiom requires that every bounded comonotonic standard sequence is finite. A related version of the Archimedean axiom has been formulated in Miyamoto (1988).

For the proof of the Theorem below Theorem VI.5.1 of [W] cannot be invoked, again, because no continuity is available. The major problem for the proof is to establish additive representability on each rank-ordered cone. This has been done in Wakker (1990c).

THEOREM 8 Let $\succeq$ be a binary relation on a Cartesian product $\mathcal{C}^{n}$. Suppose there exists a comonotonic subset with at least two essential coordinates, and restricted solvability is satisfied. Then the following two statements are equivalent:

(i) $\succeq$ maximizes CEU for a capacity $v$ on $\{1, \ldots, n\}$ and a utility function $U: \mathcal{C} \rightarrow \mathbb{R}$.

(ii) The binary relation $\succeq$ is a weak order, satisfies the comonotonic Archimedean axiom, and does not reveal comonotonic-contradictory tradeoffs.

Further, the capacity in Statement (i) is uniquely determined, and the utility function is an interval scale.

PROOF. First consider the implication (i) $\Rightarrow$ (ii), so suppose (i) holds. It immediately follows that $\succeq$ is a weak order. From Lemma 7 it follows that $\succeq$ does not reveal comonotoniccontradictory tradeoffs. The comonotonic Archimedean axiom follows from substitution.

As a preparation for the sequel, we say the equally spaced case applies if there exist consequences $\alpha \succ \gamma$ for which there is no $\beta$ with $\alpha \succ \beta \succ \gamma$. The other case is the densely spaced case. As shown in Wakker (1990c) for rank-ordered cones, and before for instance in KLST for full Cartesian products, in the equally spaced case the range of each additive value function can be taken as an interval within the integers (see Wakker, 1990c, Corollary 7), in the densely spaced case the range of each additive value function is dense within its convex hull (see Wakker, 1990c, Formula (16)). The latter is called denserangedness.

For the reversed implication, suppose (ii) holds. To derive are (i) and the uniqueness results. Exactly as in [W, Section VI.4], the following conditions can be derived: Com. CI, pointwise monotonicity $\left(\left[\forall i: x_{i} \succeq y_{i}\right] \Rightarrow x \succeq y\right.$ ), and comonotonic strong monotonicity $\left(x \succ y\right.$ if not only $\left(\left[\forall i: x_{i} \succeq y_{i}\right]\right)$, but also $x_{j} \succ y_{j}$ for some $j$ that is essential on a comonotonic set containing both $x$ and $y$ ). An elaborated self-contained presentation of the proof below would take much space. Hence a verbal presentation is given. It uses many parts of reasonings in other papers by the author.

First attention is restricted to a rank-ordered cone with two or more essential coordinates. For the time being the coordinates inessential on this cone are suppressed. Then the combination of pointwise monotonicity and comonotonic strong monotonicity on the rankordered cone is identical to 'monotonicity' in Wakker (1990c). It is straightforward that 
the absence of comonotonic-contradictory tradeoffs revelations by $\succeq$ implies the generalized triple cancellation condition on this rank-ordered cone, and that restricted solvability on $\mathcal{C}^{n}$ in the presence of the other conditions (mainly monotonicity) implies restricted solvability within the rank-ordered cone. According to Wakker (1990c, Theorem 3), the conditions of restricted solvability, transitivity, completeness, monotonicity, the Archimedean axiom, ${ }^{3}$ and generalized triple cancellation, imply the existence of additive value functions $\left(V_{j}\right)$ on this rank-ordered cone with inessential coordinates suppressed and with maximal and minimal acts deleted. Theorem 3 and Examples 25b and 25c in Wakker (1990c) also show that at this stage it cannot yet be excluded that the additive representation would be 'driven to infinity' at a maximal act, and 'driven to minus infinity' at a minimal act. Obviously constant additive value functions can be assigned to the inessential coordinates, resulting in additive value functions $\left(V_{j}\right)_{j=1}^{n}$ on the rank-ordered cone with maximal and minimal acts deleted. Similarly additive value functions are obtained on each rank-ordered cone with maximal $/ \mathrm{minimal}$ acts deleted, and with two or more coordinates essential. Lemma VI.8.2 in [W] shows that under the conditions holding there the additive value functions are all mutually proportional. The method of proof used there can however not be directly used here, because it makes use of the continuum structure of the range of the additive value functions; that structure presently is not available. It can however be shown that the nonrevelation of comonotonic-contradictory tradeoffs implies that for each pair of coordinates on each rank-ordered cone standard sequences are noncontradictory. To wit, suppose the equivalences $x_{-i, j} v_{i}, \alpha \sim x_{-i, j} w_{i}, \beta$ and $x_{-i, j} v_{i}, \beta \sim x_{-i, j} w_{i}, \gamma$ hold where all four acts are comonotonic, $i$ is essential on a comonotonic set containing the four acts, and $v_{i} \nsim w_{i}$. Suppose further that (with $*$ still to be determined) $y_{-k, l} s_{k}, \alpha \sim y_{-k, l} t_{k}, \beta$ and $y_{-k, l} s_{k}, \beta * y_{-k, l} t_{k}, \gamma$, with again these four acts comonotonic, $k$ essential on a comonotonic set containing the four acts, and $s_{k} \neq t_{k}$. The first two equivalences show that $\alpha, \beta, \gamma$ is (part of) a standard sequence on coordinate $j$ in some comonotonic set. The third equivalence occurs if $\alpha, \beta$ is a part of a standard sequence on coordinate $l$ in some, possibly different, comonotonic set. Now any other preference for * than $\sim$ would lead to comonotonic-contradictory tradeoffs. So indeed for each pair of coordinates on each rank-ordered cone standard sequences are noncontradictory. This gives the result of Lemma VI.8.2 of [W], i.e., after choice of common zero, proportionality of all obtained additive value functions, both those belonging to different coordinates in a same rank-ordered cone, and those belonging to different rank-ordered cones. So there exists one function, say $U$, such that each additive value function obtained thus far is a positive multiple of that function $U$. By Wakker (1990c, Corollary 6), the additive representation can be extended to the maximal and minimal acts; so we do. This can be done under preservation of proportionality, in the equally spaced case by a unit increase of the maximum over the values assigned to the nonmaximal acts at the maximal act, and an analogous unit decrease at the minimal act ('unit' is length of minimal gap in range of representing function), in the other (densely spaced) case by taking supremum/infimum. Next two nonequivalent constant ('certain') acts are fixed, as well as any two real numbers, and all additive representations are renormalized to assign the highest real number to the preferred of the two fixed constant acts, the lowest number to the dispreferred fixed constant act. By proportionality the additive representations must then assign the same value to all constant acts (the weights attached to $U$ have the same sum). $U$ and the attached weights are renormalized such that all weights sum to one. Rank-ordered cones with no essential coordinate cannot exist (see [W, Corollary VI.4.12]. On those with exactly one essential coordinate every 'starting' act is equivalent to the constant act identical to the one essential coordinate, and the $U$-value

\footnotetext{
${ }^{3}$ The comonotonic version here implies the version in Wakker, 1990c within every rankordered cone, because of monotonicity and suppression of inessential coordinates/states.
} 
of that constant act is assigned to the starting act.

Next it is shown that the normalized (thus coinciding on constant acts) additive representations within rank-ordered cones with all additive value functions proportional, represent in the right manner acts from different rank-ordered cones. For acts with certainty equivalents this is direct, as in Lemma VI.8.6 of [W]. Unfortunately, in the algebraic set-up of this paper there do not necessarily exist certainty equivalents for all acts. So we must elaborate.

The equally spaced case is discussed briefly. By Wakker (1990c, Subs. 3.1), within each rank-ordered cone an additive representation exists with same 'decision weights' on the states essential there. By restricted solvability, each rank-ordered cone has the same set of equivalence classes, hence the same number, say $k$, of essential states. The construction of the representations within rank-ordered cones in Wakker (1990c, Subs. 3.1) shows that there are $k-1$ equivalence classes strictly between two subsequent constant act-equivalence classes. They are valued the same in each rank-ordered cone. From this everything follows.

Next let us turn to the densely spaced case. First let $x$ and $y$ be such that, for an additive representation $V^{x}$ within a rank-ordered cone containing $x$, and for an additive representation $V^{y}$ within a rank-ordered cone containing $y$, we have $V^{x}(x)>V^{y}(y)$. It is an elementary exercise to show that denserangedness of the additive value functions together with monotonicity implies denserangedness of the additive representation when restricted to the constant acts. ${ }^{4}$ So, by monotonicity, for each nondegenerate interval within the convex hull of the range of the additive representation there exists a constant act that obtains a value within that interval. There must exist a constant act dispreferred to $x$ (e.g. the worst consequence of $y$ ), hence also a constant act $c$ such that $V^{x}(x)>V^{x}(c)>V^{y}(y)$. Because $V^{x}(c)=V^{y}(c)$, there must also exist a constant act $c^{\prime}$ such that $V^{x}(x)>V^{x}(c)=$ $V^{y}(c)>V^{y}\left(c^{\prime}\right)>V^{y}(y)$. It follows that $x \succ c \succ c^{\prime} \succ y$, the first preference within $x$ 's cone, the second and third within $y$ 's cone. The implication $V^{x}(x)>V^{y}(y) \Rightarrow x \succ y$ has been established. This also shows that equivalent acts get assigned the same value from all additive representations, in particular one same act gets the same value from all additive representations within all rank-ordered cones in which the act is contained. So one function $V$ can be defined, additive and representing within each rank-ordered cone, with all additive value functions proportional, and with $V(x)>V(y) \Rightarrow x \succ y$ for $x, y$ not contained within one same cone.

Next suppose $x \succ y$. By a reasoning similar to Lemma 11 in Wakker (1990c), there exists $x^{\prime}$ in the same rank-ordered cone as $x$ such that $x \succ x^{\prime}$, and still $x^{\prime} \succ y .{ }^{5}$ By the results of the previous paragraph $V(x)>V\left(x^{\prime}\right) \geq V(y)$ follows.

The results of the last two paragraphs show that $V$ is representing. That $V$ is a Choquet integral is now demonstrated similarly to Lemma VI.8.7 and Theorem VI.8.8 (now including maximal and minimal consequences) of [W].

The standard uniqueness results follow from the uniqueness results for the additive representations on the rank-ordered cones without the maximal/minimal acts as obtained in Theorem 3 of Wakker (1990c), then by proportionality extend to these maximal and minimal acts after all, and for the remainder are similar to [W, Theorem VI.5.1].

\footnotetext{
${ }^{4}$ E.g., for any nonmaximal constant act $c$ and any $\epsilon>0$ there exists by monotonicity another constant act $c^{\prime} \succ c$ such that all additive value functions assign to $c^{\prime}$ a value exceeding the value of $c$ by less than $\epsilon / n$.

${ }^{5}$ Say the cone has first coordinate best, second coordinate second-best, and so on. Take the nonminimal coordinate $x_{j}$ of $x$ with $j$ essential within the cone and as high as possible. Decrease (= change into a less preferred consequence) the subsequent nonessential $x_{j+1}, x_{j+2}, \ldots$ equivalent to $x_{j}$. This does not affect the equivalence class of the act. Next decrease $x_{j}$ as in Lemma 11 of Wakker (1990c).
} 


\section{Remaining Results in [W], and Conclusion}

In Chapter $\mathrm{V}$ in $[\mathrm{W}]$ the representation of SEU as in Theorem 5 has been extended to infinite state spaces. A similar adaptation could be obtained for the algebraic approach. The assumption of the existence of a certainty-equivalent is needed for the method that we have in mind though; for the algebraic set-up that is really an additional restrictive assumption. A more general set-up for infinite state spaces and unbounded utilities has been given in Wakker (1989c), dealing also with CEU.

The first part of Chapter VII of [W] deals with convex/concave additive representations on mixture spaces. These will lead to continuums of ranges of additive representations, so that the topological approach is not more restrictive than the algebraic. The second part of Chapter VII of [W] deals with risk aversion and real numbers (amounts of money) as consequences. Here strict increasingness of utility is a natural condition. This rules out countability of the range of utility, thus the equally spaced case, so leads to the densely spaced case. That excludes 'jumps' of $U: U$ is continuous after all. Again, here the algebraic approach does not generalize the topological approach.

For the other major conjoint measurement results of [W], this paper has given an essential generalization, of the topological approach to the algebraic approach. That this is a desirable extension has been argued in Wakker (1988).

\section{REFERENCES}

Anscombe, F.J. \& R.J. Aumann (1963). A Definition of Subjective Probability. Annals of Mathematical Statistics, 34, 199-205.

Armstrong, T.E. (1990). Comonotonicity, Simplicial Subdivision of Cubes and NonLinear Expected Utility via Choquet Integrals. Dept. of Mathematics and Statistics, University of Maryland, Baltimore, Maryland 21228.

Choquet, G. (1953-4). Theory of Capacities. Annales de l'Institut Fourier 5 (Grenoble), $131-295$.

de Finetti, B. (1931). Sul Significato Soggettivo della Probabilità. Fundamenta Mathematicae, 17, 298-329.

de Finetti, B. (1937). La Prévision: Ses Lois Logiques, ses Sources Subjectives. Annales de l'Institut Henri Poincaré 7, 1-68. Translated into English by H.E. Kyburg, Foresight: Its Logical Laws, its Subjective Sources. In H.E. Kyburg \& H.E. Smokler (1964, Eds.), Studies in Subjective Probability. New York: Wiley, 53-118.

de Finetti, B. (1974). Theory of Probability, Vol.I. New York: Wiley.

Fishburn, P.C. (1970). Utility Theory for Decision Making. New York: Wiley.

Fishburn, P.C. (1990), 'Skew Symmetric Additive Utility with Finite States', Mathematical Social Sciences 19, 103-115.

Gilboa, I. (1987). Expected Utility with Purely Subjective Non-Additive Probabilities. Journal of Mathematical Economics, 16, 65-88.

KLST $=$ Krantz, Luce, Suppes, \& Tversky (1971) 
Krantz, D.H., R.D. Luce, P. Suppes, \& A. Tversky (1971) (=KLST). Foundations of Measurement, Vol. I. (Additive and Polynomial Representations). New York: Academic Press.

Luce, R.D. (1988). Rank-Dependent, Subjective Expected-Utility Representations. Journal of Risk and Uncertainty, 1, 305-332.

Miyamoto, J.M. (1988). Generic Utility Theory: Measurement Foundations and Applications in Multiattribute Utility Theory. Journal of Mathematical Psychology, 32, $357-404$.

Moulin, H. (1988). Axioms of Cooperative Decision Making. New York: Cambridge.

Nakamura, Y. (1990a). Subjective Expected Utility with Non-Additive Probabilities on Finite State Space. Journal of Economic Theory, forthcoming.

Nakamura, Y. (1990b). Multi-Symmetric Structures and Non-Expected Utility, Discussion paper. Inst. Socio-Econ. Plann., University of Tsukaba.

Pfanzagl, J. (1968). Theory of Measurement. Vienna: Physica-Verlag.

Pratt, J.W., H. Raiffa, \& R. Schlaifer (1964). The Foundations of Decision under Uncertainty: An Elementary Exposition. Journal of American Statistical Association, 59, $353-375$.

Ramsey, F.P. (1931). Truth and Probability. In The Foundations of Mathematics and other Logical Essays, 156-198, Routledge and Kegan Paul, London. Reprinted in H.E. Kyburg \& H.E. Smokler (1964, Eds.), Studies in Subjective Probability, New York: Wiley, 61-92.

Savage, L.J. (1954). The Foundations of Statistics. New York: Wiley. (Second edition 1972, New York: Dover.)

Schmeidler, D. (1989). Subjective Probability and Expected Utility without Additivity. Econometrica, 57, 571-587.

Suppes, P. (1956). The Role of Subjective Probability and Utility in Decision Making. Proceedings of the Third Berkeley Symposium on Mathematical Statistics and Probability, 1954-1955, 5, 61-73.

von Neumann, J. \& O. Morgenstern $(1944,1947,1953)$. Theory of Games and Economic Behavior. Princeton NJ: Princeton University Press.

$[\mathrm{W}]=$ Wakker $(1989 \mathrm{~b})$.

Wakker, P.P. (1984). Cardinal Coordinate Independence for Expected Utility. Journal of Mathematical Psychology, 28, 110-117.

Wakker, P.P. (1986). Representations of Choice Situations. Ph.D. Dissertation, University of Brabant, Department of Economics, The Netherlands.

Wakker, P.P. (1988). The Algebraic Versus the Topological Approach to Additive Representations. Journal of Mathematical Psychology, 32, 421-435.

Wakker, P.P. (1989a), 'Transforming Probabilities without Violating Stochastic Dominance'. In E.E.Ch.I. Roskam (Ed.), Mathematical Psychology in Progress, Springer, Berlin, 29-47.

Wakker, P.P. (1989b) (=[W]). Additive Representations of Preferences, A New Foundation of Decision Analysis. Dordrecht: Kluwer Academic Publishers. 
Wakker, P.P. (1989c). From Finite- to Infinite-Dimensional Integral Representations; Unbounded Utility for Savage (1954) and Others. Mathematics of Operations Research, accepted subject to revision.

Wakker, P.P. (1990a). A Behavioral Foundation for Fuzzy Measures. Fuzzy Sets and Systems, 37, 327-350.

Wakker, P.P. (1990b). Characterizing Optimism and Pessimism Directly through Comonotonicity. Journal of Economic Theory, 52, 453-463.

Wakker, P.P. (1990c). Additive Representations of Preferences on Rank-Ordered Subsets of Cartesian Products; Part I: The Algebraic Approach. Journal of Mathematical Psychology, forthcoming.

Wakker, P.P. (1991). Additive Representation for Equally-Spaced Structures. Journal of Mathematical Psychology, 35, 260-266.

Yaari, M.E. (1987). The Dual Theory of Choice under Risk. Econometrica, 55, 95-115. 